\title{
Animal study of Anthracycline-induced Cardiotoxicity and Nephrotoxicity and Evaluation of Protective Agents
}

\author{
Amani N. Shafik ${ }^{1}$, Mostafa M. Khodeir ${ }^{2 *}$ and Mostafa S. Fadel ${ }^{3}$
}

${ }^{1}$ Assistant professor, Department of Pharmacology, Faculty of Medicine, Cairo University, Egypt

${ }^{2}$ Lecturer, Department of Pathology, Faculty of Medicine, Cairo University, Egypt

${ }^{3}$ Department of ultrasonography, Animal Reproduction Research Institute, Agricultural Research Center, Egypt

\begin{abstract}
Study background: Anticancer chemotherapy Anthracycline (ANT) antibiotics associated with cardiac and renal toxicity which represents serious complication. In this study, the protective effect of the 2 beta-blockers carvedilol and nebivolol were tested in a rat model of ANT induced cardio and renal-toxicity by repeated intraperitoneal doxorubicin (Dox) administration.

Methods: Six groups of animals were used, each 8 rats divided as control group- received intraperitoneal saline every other day; control carvedilol: rats received carvedilol dose $30 \mathrm{mg} / \mathrm{kg} /$ day orally; control nebivolol: rats received nebivolol $1 \mathrm{mg} / \mathrm{kg} /$ day orally; doxorubicin alone: a dose of $3 \mathrm{mg} / \mathrm{kg} /$ day was administered intraperitoneal every other day; doxorubicin + carvedilol: carvedilol started at the same day with Dox; doxorubicin + nebivolol: nebivolol started at the same day with Dox. All substances were administered for 12 days. Detection \& quantification of doxorubicin-induced heart and renal damage and therapeutic action of beta-blockers was done using Langendorff's technique, echo-cardiographic function examinations, serum creatinine, total proteins and histopathology.
\end{abstract}

Results: The administration of doxorubicin in the dose of $3 \mathrm{mg} / \mathrm{kg} /$ day for 12 days produced pronounced heart impairment, as well as renal nephrotoxic changes. Significant reduction of Doxo-cardiotoxicity and nephrotoxicity in Nebivolol-treated animals more than Carvedilol treated animals.

Conclusions: Coadministration of either carvedilol or nebivolol with doxorubicin was able to ameliorate up to almost contradict doxorubicin-induced myocardial injury, glomerular filtration disturbance and renal tubular damage with upper hand for nebivolol. So, they can be considered a feasible candidate to protect against nephrotoxicity \& cardiotoxicity commonly encountered with doxorubicin treatment.

Keywords: Anthracycline; Toxicity; Nebivolol; Carvedilol

\section{Introduction}

Doxorubicin (Dox) is one of the most potent broad-spectrum antitumor anthracycline antibiotics, widely used to treat a variety of cancers including breast, ovarian, lung, uterine and cervical cancers, Hodgkin's disease, non-Hodgkin lymphoma, acute leukemia, soft tissue and primary bone sarcomas [1-3]. Because of their great importance in cancer therapy, researchers have expended great efforts trying to prevent or attenuate the side effects of Dox administration. Accordingly, several approaches have been pursued in the 90 's, such as dosage optimization, synthesis, and use of analogues or combined therapy. No hopeful results have been found and the application of different Dox-analogues did not show better antineoplastic value or lower toxicity than Dox $[4,5]$.

Many different chemical agents have been examined to prevent ANT-induced cytotoxicity $[4,6]$, and some of them showed promising results. These chemicals includes natural Products e.g. the most commonly used and investigated compounds are vitamins (E, C, A, carotenoids), coenzyme Q, flavonoids, polyphenols, herbal antioxidants, selenium, and virgin olive oil [7].

Some well-known as well as new and potential drugs have been shown to have a significant influence on Dox-induced toxicity throughout different pathways of protection. Stolarska et al. [8] investigated the cytoprotective effect of amifostine against cardiotoxicity due to Dox in the treatment of childhood neoplastic diseases. Doxycycline protection against Dox-induced oxidative stress and apoptosis in mouse testes was observed by Yeh et al. [9]. Liu et al. [10] have compared the roles of dihydropyridine calcium antagonists: nifedipine, nitrendipine, and amlodipine in Dox-induced nephrotoxicity. Published in vivo studies suggested that clinically available cytokine "erythropoietin" may play a protective role against nonhematopoietic diseases, including cardiotoxicity induced by Dox, infarction and ischemia-reperfusion injury in the heart [11]. Pre-administration of docetaxel protects against Dox-induced cardiotoxicity [12]. Fullerenols act as a free radical scavenger in biological systems has repeatedly demonstrated protective effects against cardio-, hepato- and nephrotoxicity induced by Dox in animal models $[13,14]$.

Despite extensive research, the mechanism of Dox-induced toxicity is still not completely elucidated and neither are the mechanisms of the different chemicals acting as potential protectors. Clinical trials and further studies are warranted to investigate and compare the individual mechanisms of beneficial effects [15].

The third generation of $\beta$-adrenoreceptor antagonists including nebivolol and carvedilol represents a distinct pharmacological entity as compared with other $\beta$-adrenoreceptor antagonists $[16,17]$.

*Corresponding author: Mostafa Mahmoud Khodeir, 8 B el Malek el Nase Street, Khayrat Street, Lazoughli 0106382137; E-mail: dr_mustafa31@yahoo.com Received February 21, 2011; Accepted March 24, 2011; Published May 15, 2011 Citation: Shafik AN, Khodeir MM, Fadel MS (2011) Animal study of Anthracyclineinduced Cardiotoxicity and Nephrotoxicity and Evaluation of Protective Agents. Cancer Sci Ther 3: 096-103. doi:10.4172/1948-5956.1000068

Copyright: ( 2011 Shafik AN, et al. This is an open-access article distributed unde the terms of the Creative Commons Attribution License, which permits unrestricted use, distribution, and reproduction in any medium, provided the original author and source are credited. 
Carvedilol is a cardiovascular drug with a wide therapeutic potential. It is a non selective blocker of $\alpha 1$ and $\beta$-adrenergic receptors, while displaying vasodilating properties has potent antioxidant and antiapoptotic properties $[18,19]$.

In addition to the inhibitory effects on $\beta 1$-adrenergic receptors, the novel $\beta$-blocker nebivolol has additional hemodynamic properties [20]. In particular, nebivolol is able to stimulate endogenous production of NO by inducing phosphorylation of the endothelial enzyme endothelial NO synthase (NOS; eNOS) [21]. Whereas nebivolol action on NO can result in favorable outcomes at vascular levels, the effects of $\mathrm{NO}$ on the heart are not so straightforwardly beneficial.

We designed in this study to establish the effect of Administration of doxorubicin in combination with either carvedilol or nebivolol Betablocker agents in order to block its free-radical mediated cardio- and nephro-toxic effect, thus reducing its cardiotoxicity and nephrotoxicity.

\section{Materials and Methods}

-Doxorubicin Hydrochloride (DOX): (Pharmacia and Upjohn. Italy).

$\mathrm{C}_{27} \mathrm{H}_{29} \mathrm{NO}_{11}$, HCL. DOX was supplied as a red-orange, hygroscopic, crystalline powder. DOX solution was freshly prepared, protected from light. It was dissolved in saline solution.

-Nebivolol powder (Sigma chemical company). Nebivolol was dissolved in distilled water.

-Carvedilol powder (Sigma chemical company). Carvedilol was dissolved in distilled water.

-Isoprenaline powder (Sigma chemical company). Isoprenaline was dissolved in distilled water.

-Modified Krebs-Henseleit buffer solution of the following composition: $\mathrm{NaCl}, 118 \mathrm{mmol} / \mathrm{L} ; \mathrm{KCl}, 4.7 \mathrm{mmol} / \mathrm{L} ; \mathrm{CaCl}, 2.0$ $\mathrm{mmol} / \mathrm{L} ; \mathrm{MgSO} 47 \mathrm{H} 2 \mathrm{O}, 1.2 \mathrm{mmol} / \mathrm{L} ; \mathrm{KH} 2 \mathrm{PO} 4,1.2 \mathrm{mmol} / \mathrm{L}$; glucose, $11.1 \mathrm{mmol} / \mathrm{L}$; and NaHCO3, $25 \mathrm{mmol} / \mathrm{L}$ [22]. All chemicals from Sigma chemical company.

\section{Creatinine kit. DIAMOND}

\section{-Total proteins kit. Bio-Analytics}

-PowerLab Data Acquisition and Analysis Systems for Monitoring and recording signals from the isolated rabbit's heart connected to the Langendorff"s coronary perfusion set.

-We used 48 male albino rats, of the same age and weight 250-300 g. They were housed under standard laboratory conditions in the animal house of Research Institute of Ophthalmology.

The animals were placed every 2 in a cage, fed a standard rat chow diet and given water ad libitum under a $12 \mathrm{hr}$ light/dark cycle and room temperature. Rats were acclimatized to the environment for one-week prior to experimental use.

This study was approved by our institution's (kasr el eini hospital) Animal Care Committee and the guidelines were strictly adhered to. each):

-Rats were randomly divided in six experimental groups (8 rats

1st group - control saline: treated with $0.5 \mathrm{ml}$ of $0.9 \% \mathrm{NaCl} /$ day intraperitoneal every other day. 2nd group - control carvedilol: rats treated with carvedilol dose $30 \mathrm{mg} / \mathrm{kg} /$ day (in $0.5 \mathrm{ml}$ solution) [23] by oral gavage for 12 days.

3rd group - control nebivolol: rats treated with nebivolol dose $1 \mathrm{mg} / \mathrm{kg} /$ day [24] by oral gavage for 12 days.

4th group - Dox alone: a dose of $3 \mathrm{mg} / \mathrm{kg} / \mathrm{day}(18 \mathrm{mg} / \mathrm{kg}$ cumulative total dose) was administered intraperitoneally (in $0.5 \mathrm{ml}$ solution) every other day during12 days of the experiment [25].

5th group - Dox + carvedilol: rats treated with carvedilol dose $30 \mathrm{mg} / \mathrm{kg} /$ day by oral gavage for 12 days started at the same day with dox.

6th group - Dox + nebivolol: rats treated with nebivolol dose $1 \mathrm{mg} / \mathrm{kg} /$ day by oral gavage for 12 days started at the same day with dox.

\section{At the end of the experiment, the animals were subjected to:}

1. Blood samples collection after $18 \mathrm{hr}$ fast, from the retro-orbital sinus for determination of: Serum creatinine by the kinetic method according to Menry [26]; while total proteins were measured according to the method of Reinhold [27].

2. Echocardiography was done at the end of the experiment under light ether anesthesia [28].

Using (My lab 30VET Gold) of Esaote company, the echocardiography was used to evaluate the cardiac functions. Echocardiograms were performed with an echocardiography system equipped with a 4-8 $\mathrm{MHz}$ phased-array probe (PA122E) placed over the left parasternal area and rocked through the heart from the apex to the base. Two- dimensional short axis view of the left ventricle and M-mode tracings were recorded through the anterior and posterior left ventricular (LV) walls at the papillary muscle level to measure LV enddiastolic dimension (LVEDD) and LV end-systolic dimension (LVESD); also ejection fraction (EF) and fractional shortening (FS) were recorded. $\mathrm{FS}=$ (End-diastolic dimension -End-systolic dimension) $/$ End-diastolic dimension $\times 100 \%$.

3. Isolated perfused rat heart. Langendorff's technique [29]. The rat hearts were obtained for detection of cardiac contractile strength (inotropic effects) and heart rate (chronotropic effects) using a Power Lab acquisition system.

Rats were injected intraperitonealy (i.p.) with heparin sodium 5000 I.U/kg. They were anesthetized using ether inhalation and then sacrificed. The heart was rapidly excised and placed in icecold Krebs-Henseleit buffer bubbled constantly with 95\% O2 and $5 \%$ CO2. Perfusate temperature was maintained at $37^{\circ} \mathrm{C}$. The heart was immediately mounted and cannulated in the retrograde mode according to Langendorff method as previously described [29]. The amplitude of ventricular contractions as well as heart rates of different groups was recorded continuously on a Power Lab acquisition system (ADInstruments Pty Ltd, Castle Hill, Australia). Concentrationresponse curves to isoprenaline $(\mu \mathrm{g} / \mathrm{ml})$ were constructed to study the inotropic and chronotropic effects.

4. Sections from heart and kidneys were obtained and fixed in 10\% neutral phosphate-buffered formalin, dehydrated in ethanol, cleared in xylene and embedded in paraffin. Five-micrometer sections were stained with Hematoxylin and eosin for histopathological examination.

Another set of heart sections were stained by toluidine blue to be 
Citation: Shafik AN, Khodeir MM, Fadel MS (2011) Animal study of Anthracycline-induced Cardiotoxicity and Nephrotoxicity and Evaluation of Protective Agents. J Cancer Sci Ther 3: 096-103. doi:10.4172/1948-5956.1000068

graded for cardiotoxicity. Histopathological changes, including loss of myofibrils and vacuolization of the cytoplasm are used to grade injury on a scale of 1 to 3 . Fewer than $5 \%$ of cells show changes of injury are
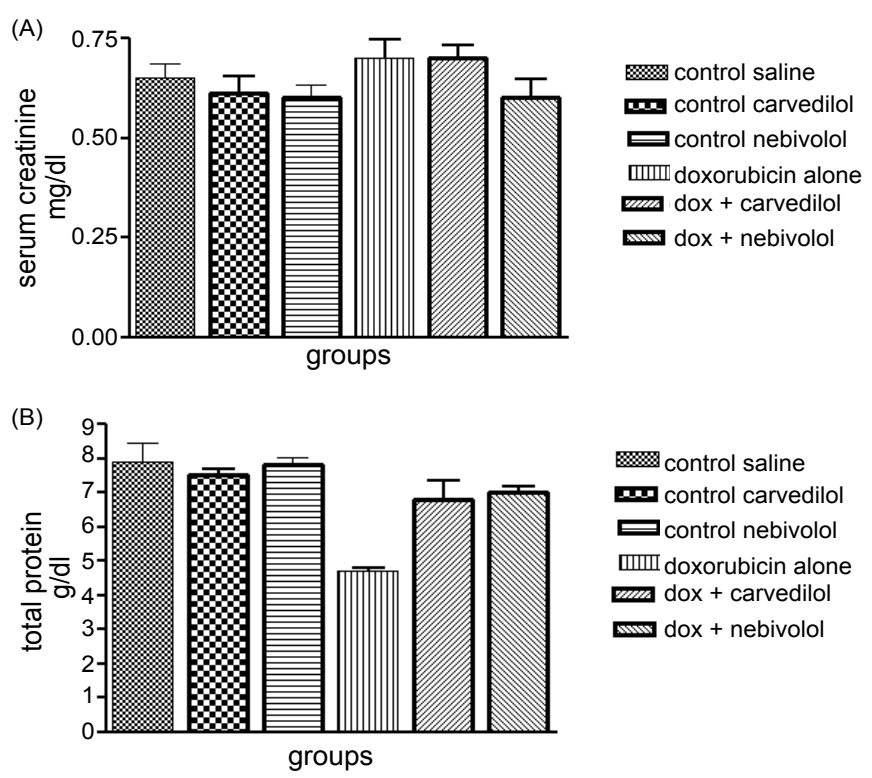

Figure 1: Serum levels of: A. Creatinine (mg/dl). B. Total Proteins $(\mathrm{g} / \mathrm{dl})$.
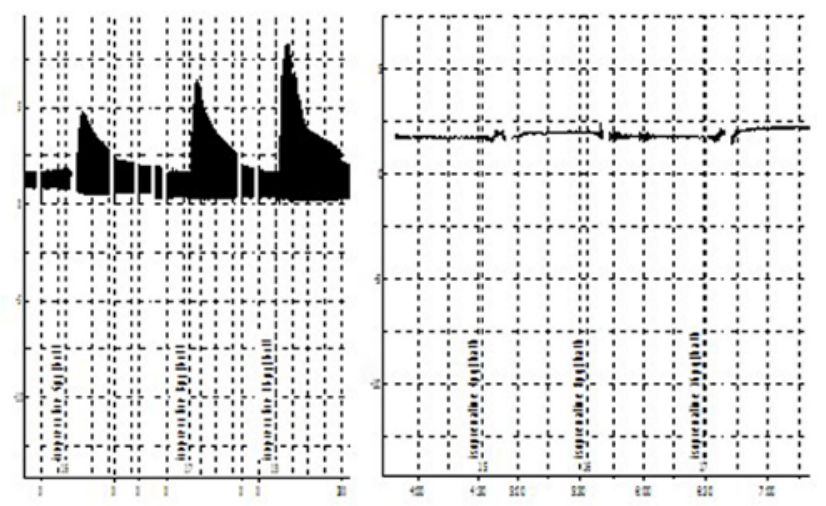

A.

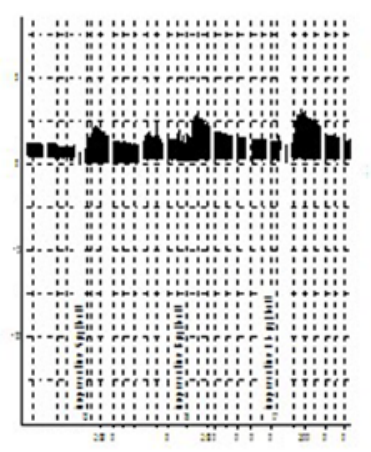

B.

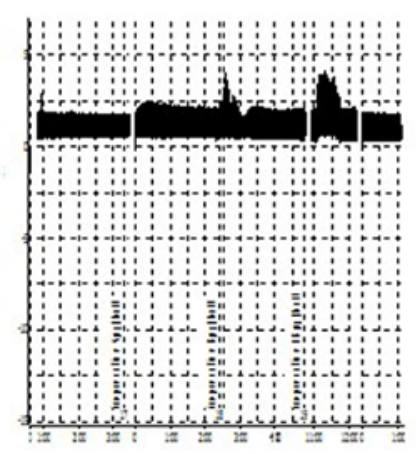

D.

Figure 2: Changes in Power of cardiac contractility and heart rate in response to increasing concentrations of isoprenaline $(\mu \mathrm{g} / \mathrm{ml})$ recorded by Langendorff-perfused rat hearts. A. Normal untreated rat B. Doxorubicin treated rat. C.Dox + carvedilol treated rats D. Dox + nebivolol treated rats.

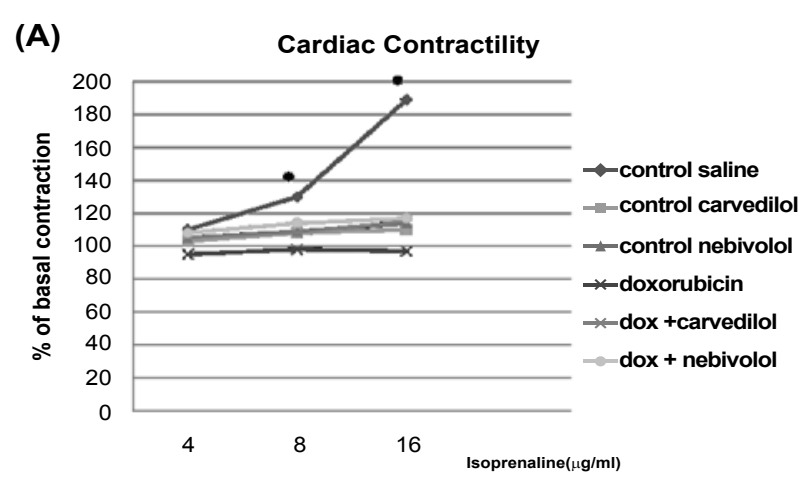

*Significant difference from the basal contractivity-p $<0.05$

(B)

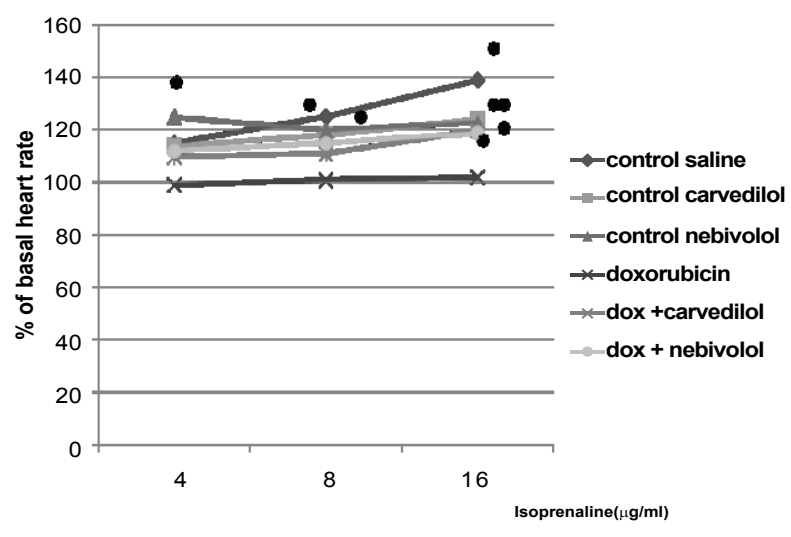

*Significant difference from the basal heart rate-p $<0.05$

Figure 3: Changes in Power of cardiac contractility and heart rate in response to increasing concentrations of isoprenaline, calculated as percentage of baseline (\%). A. Cardiac contractility B. Heart rate.

given a grade 1 .Those with changes in 5 to $15 \%$ graded 1.5 . While 16 to $25 \%$ were graded 2. 26 to $35 \%$ graded 2.5. Finally, More than $35 \%$ of cells were graded 3 [30].

\section{Statistical analysis}

The Stat Plus ${ }^{\circledR}$ program was used for the statistical evaluation; values were compared by an unpaired $t$ - test. Values with $\mathrm{p}<0.05$ were considered significant.

\section{Results}

\section{Serum level of creatinine}

Dox-alone group showed statistical significant rise in creatinine levels compared to control groups $(\mathrm{p}<0.05)$. Dox-nebivolol treated group showed significant decrease in levels of creatinine compared to dox alone group ( $p=0.0006)$, also it showed significant lower creatinine levels compared to dox-carvedilol treated group $(\mathrm{p}=0.0002)$ (Figure 1a).

\section{Total proteins}

Doxorubicin therapy was shown to decrease total proteins significantly after 12 days as compared to the control values $(\mathrm{p}<0.0001)$. Treatment with both carvedilol and nebivolol significantly increased the total proteins concentration compared to dox alone group $(\mathrm{p}<0.0001)$, with insignificant difference between the two drugs. However, the concentrations of total proteins are still significantly lower than the control $(\mathrm{p}<0.05)$ (Figure $1 b)$. 
Citation: Shafik AN, Khodeir MM, Fadel MS (2011) Animal study of Anthracycline-induced Cardiotoxicity and Nephrotoxicity and Evaluation of Protective Agents. J Cancer Sci Ther 3: 096-103. doi:10.4172/1948-5956.1000068

\section{Echocardiography (Table 1)}

LVSD and LVDD were significantly dilated $(\mathrm{p}<0.0001)$ in doxuntreated rats, compared to control groups, whereas a significant reduction in both end-diastolic and -systolic volume was observed in both carvedilol and nebivolol treated dox rats compared to dox untreated rats $(\mathrm{p}<0.05)$. Also, nebivolol treated dox rats showed significant reduction compared to dox-carvedilol group $(\mathrm{p}<0.0001)$.

LV EF values were decreased during the experiment in Doxreceiving animals-from $92 \pm 1.9 \%$ in the control to $63 \pm 2.9 \%$ at the end of the experiment, the decrease being statistically significant $(\mathrm{p}<0.0001)$.

In the carvedilol and nebivolol treated control groups, the LV EF values did not differ significantly from the control group. While in the carvedilol and nebivolol treated dox groups, the LV EF values were not significantly decreased as compared to control groups but significantly higher than dox treated group $(\mathrm{p}<0.0001)$.

Percentage fractional shortening (\%FS), as derived from the measurements on M-mode echocardiogram, were not significantly different in the three control groups. However, there was a significant reduction at 12 days in dox-untreated rats compared with the other groups $(\mathrm{p}<0.05)$.

\section{Isolated perfused rat heart preparation (Langendorff's preparation)}

Isoprenaline produced concentration-dependent increase in force and heart rat. The positive inotropic and chronotropic effects of isoprenaline were not significantly different in hearts from the two treated control groups of rats (Figure 2 and 3). However, the positive inotropic and chronotropic effects induced by isoprenaline was significantly reduced $(\mathrm{P}<0.05)$ in hearts from the dox group compared to normal untreated and control treated rats $(\mathrm{p}<0.05)$ (Figure 2$)$. Both dox treated groups (carvedilol and nebivolol) showed significant improvement in power of cardiac contractility and heart rate (inresponse to increasing doses of isoprenaline) (Figure 3) compared to dox alone group $(\mathrm{p}<0.05)$. There were insignificant change between the Dox + carvedilol and Dox + nebivolol groups $(\mathrm{p}>0.05)$ when compared to each other (Table 2).

\section{Histopathological assessments}

Heart: There was no abnormal microscopy for the cardiac muscles of all three control groups in the light microscopic examination. Doxorubicin alone group showed 3 as highest recorded grade of cardiac muscle injury (Figure $4 \mathrm{~A}$ ). In dox + carvedilol treated rats group the highest grade recorded was 1.5 which was much lower than those of doxorubicin alone group (Figure 4B). While, dox + nebivolol treated rats groups showed lowest grade of cardiac injury which was 1 (Figure $4 \mathrm{C})$. Both treatment groups showed statistical significant difference compared to the Dox alone group $(\mathrm{p}<0.0001)$. Again, dox + nebivolol treated group showed statistical significance difference compared to dox + carvedilol group $(\mathrm{p}=0.03)$.

Kidneys: There was no abnormal microscopy for the kidney of all three control groups in the light microscopic examination. On the other hand, light microscopic examination of kidneys' rats of doxorubicin alone group revealed glomerulopathy characterized by mild hyperplasia of mesangium, and moderate tubular atrophy and dilation. Marked albumin (hyaline) casts formation and congestion of interstitial capillaries (Figure 5). In dox + carvedilol treated rats group shows mild increase in mesangium, focal tubular degeneration and dilation. Mild albumin (hyaline) casts formation. In dox + nebivolol treated rats groups revealed only minimal albumin casts formation.

\section{Discussion}

The present study shows a prominent cardio and renal-protection elicited by nebivolol, and, to a lesser degree carvedilol in a rat model of doxorubicin-mediated toxicity. Anthracyclines have gained widespread use in the treatment of haematological malignancies and solid tumours, but their cumulative toxicity on the many organs prevents their use at their maximum myelotoxic doses during the optimal number of therapeutic cycles required[31].

Doxorubicin $18 \mathrm{mg} / \mathrm{kg}$ cumulative total dose - induced cardiomyopathy in the form of impaired LV EF, fractional shortening, LVDD and LVSD dilatation; marked impaired contractility with decease in heart rate, with failure of $\beta$-adrenergic response to isoprenaline together with cardiac muscle injury.

Calcium homeostasis disturbances related to the appearance of oxidative stress could explain why the response to isoprenaline was attenuated in the dox-treated group. The dysfunction of sarcoplasmic reticulum could induce changes in intracellular calcium concentrations [32], leading to impairment of cardiac contractility and/or relaxation. Moreover, it has been reported that anthracycline treatment could induce the reduction of $\beta$-adrenergic receptor density on myocardial membrane [33].

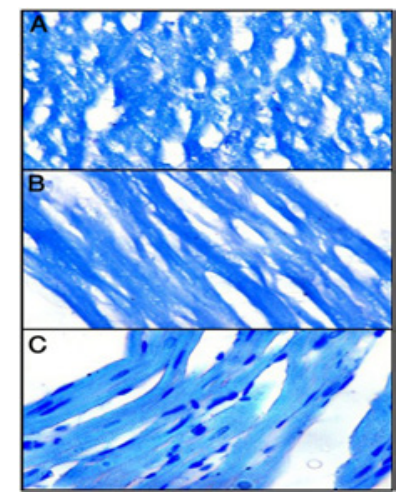

Figure 4: Different sections of rat's hearts with Toluidine blue stain (x1000): A: Doxorubicin untreated group showed marked loss of myofibrils and vacuolization of cytoplasm. B: Doxorubicin-Carvedilol treated group showed partial preservation of myofibrils and moderate cytoplasmic vacuolization.C: Doxorubicin- Nebivolol treated group showed best preservation of myofibrils and minimal vacuolization of cytoplasm.

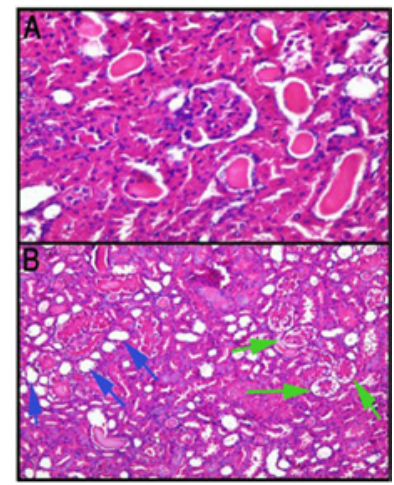

Figure 5: Sections in rat kidneys of Doxorubicin untreated group showing. A: Mild increase of mesangium and marked hyaline casts formation (Hematoxylin \&eosin x400). B: Areas of tubular hydropic degeneration (Green arrows) \& tubular atrophy (Blue arrows) (Hematoxylin \&eosin x100). 
Citation: Shafik AN, Khodeir MM, Fadel MS (2011) Animal study of Anthracycline-induced Cardiotoxicity and Nephrotoxicity and Evaluation of Protective Agents. J Cancer Sci Ther 3: 096-103. doi:10.4172/1948-5956.1000068

\begin{tabular}{|c|c|c|c|c|}
\hline \multirow[b]{2}{*}{ Groups } & \multicolumn{4}{|l|}{ Echocardiographic Cardiac Functions } \\
\hline & LV end-systolic dimension (LVSD) (mm) & LV end-diastolic dimension (LVDD) (mm) & Ejection fraction (EF) (\%) & Fractional shortening (FS) (\%) \\
\hline control saline & $2.8 \pm 0.015$ & $8.9 \pm 0.01$ & $92 \pm 1.9$ & $59 \pm 1.3$ \\
\hline control carvedilol & $3 \pm 0.03$ & $7 \pm 0.015$ & $90 \pm 2$ & $56 \pm 1.4$ \\
\hline control nebivolol & $3.2 \pm 0.012$ & $6.8 \pm 0.02$ & $89 \pm 1.6$ & $55 \pm 1.1$ \\
\hline doxorubicin alone* & $9 \pm 0.05$ & $12 \pm 0.15$ & $63 \pm 2.9$ & $30 \pm 1.5$ \\
\hline dox + carvedilolt & $4 \pm 0.02$ & $8.8 \pm 0.025$ & $80 \pm 1.7$ & $51 \pm 1.3$ \\
\hline dox + nebivolol $\ddagger \S$ & $4.7 \pm 0.014$ & $8.6 \pm 0.016$ & $88 \pm 2$ & $45 \pm 1.6$ \\
\hline
\end{tabular}

${ }^{*}$ All measures of Dox alone group significantly different from control groups $p<0.0001$

† All measures of Dox-Carvedilol group was significantly different from Dox alone group $p<0.05$

$\ddagger$ All measures of Dox-Nebivolol group was significantly different from Dox alone group $p<0.0001$

$\S$ All measures of Dox-Nebivolol group was significantly different from Dox-Carvedilol group $p<0.05$

Table 1: Effect of carvedilol and nebivolol on Doxorubicin induced Cardiac Echocardiographic changes. Values are expressed as mean \pm S.D. ( $n=8$ )

\begin{tabular}{|l|l|l|}
\hline Groups & Power of cardiac contractility $(\mathrm{gm})$ & Heart rate $(\mathrm{bpm})$ \\
\hline control saline & $3.778 \pm 0.7104$ & $326.2 \pm 18.59$ \\
\hline control carvedilol & $2.579 \pm 0.4133$ & $231.3 \pm 19.04$ \\
\hline control nebivolol & $2.658 \pm 0.3995$ & $235.7 \pm 14.94$ \\
\hline doxorubicin alone* & $1.433 \pm 0.2861$ & $75.33 \pm 12.09$ \\
\hline dox + carvedilol† & $2.170 \pm 0.2445$ & $258.5 \pm 28.06$ \\
\hline dox + nebivolol $§$ & $2.071 \pm 0.2263$ & $250.3 \pm 29.93$ \\
\hline
\end{tabular}

*All measures of dox alone group was significantly different compared to control groups $p<0.0001$

$\dagger$ Cardiac contractility and heart rate were significantly different in dox-carvedilol treated group compared dox alone group $p=0.0001 \&<0.0001$ respectively

$\ddagger$ dox-nebivolol treated group showed significant difference in cardiac contractility and heart rate compared to dox alone group $p=0.0002 \&<0.0001$ respectively

$\S$ No significant difference between measures of dox-nebivolol and dox-carvedilol treated groups $p>0.05$

Table 2: Cardio dynamic Measurements in Langendorff-perfused rat hearts. Data are presented as mean \pm S.D. $(n=8)$.

This is in agree with Filomena de Nigrisa et al. [34] who evaluated the cardiac toxicity of ANTs by the left ventricular pressure developed under a constant perfusion pressure (LVDP), the rate of variation of this parameter during systole (contractility) (LV/dt)max and during diastole (relaxation) (LV(dP/dt)min. Also Paulo et al. [35] confirmed that DOX induces oxidative stress, mitochondrial dysfunction, and histopathological lesions in the cardiac tissue in DOX-induced mitochondrial-mediated cardiomyopathy.

Also, Doxorubicin-induced glomerulopathy is evident by decrease in both serum creatinine and total proteins, associated with swelling and vacuolation of epithelial cells, moderate tubular atrophy and dilation; marked albumin (hyaline) casts formation and congestion of interstitial capillaries. These results are consistent with previous studies reported by other investigators [36,37] that doxorubicin induced nephrotoxicity in normal rats.

According to the reported results for Dox-induced toxicity, almost all organs can be attacked and damaged via the formation of free oxygen radicals and some of the organo-specific pathways. Several mechanisms have been proposed to account for Dox cardiotoxicity, e.g., free radical stress, calcium overloading, and mitochondrial dysfunction $[38,39]$.

The exact mechanism of doxorubicin-induced nephrotoxicity is not yet known. However, it has been suggested by many investigators that cellular damage induced by doxorubicin is mediated by the formation of an iron anthracycline free radical, which in turn causes severe damage to the plasma membrane [40].

Administration of either carvedilol or nebivolol alone in normal rats did not result in significant reduction of normal heart rate in isolated perfused langendorff heart.

Chronic administration of beta-blockers without additional effects produces reactive up-regulation of beta-receptor density [41]. In addition, beta-blockers reduce nocturnal melatonin production [42]. Indeed, under conditions with a physiologically low sympathetic tone, i.e. at rest and during recovery, carvedilol failed to significantly decrease heart rate.

The beneficial effect of beta blockers (BBs) on anthracycline induced toxicity, they exert a significant protective effect on the cardiac and renal toxicity induced by doxorubicin in this rat model. Coadministration of either carvedilol or nebivolol with doxorubicin was able to ameliorate up to almost contradict doxorubicin-induced myocardial injury, glomerular filtration disturbance and renal tubular damage with upper hand for nebivolol.

Recent developments in cell biology allow us to understand that not all BBs are equal, as their intracellular mechanisms of action can be very different [43].

A study by Filomena de Nigrisa et al. [34] show a beneficial effect of BBs on anthracycline treated hearts. In particular, the use of nebivolol or carvedilol with anthracyclines have reduced the release of glutathione (GSSG) and reduced glutathione (GSH). Since the most important property of carvedilol is antioxidative profile and nebivolol predominantly effects nitric oxide (NO) pathway, the common protective pathway of nebivolol and carvedilol could be the intrinsinc beta blocker properties coupled to antioxidant and NO release. Previous studies showed that patients treated with $\beta 1$-selective antagonists without intrinsic sympathomimetic activity (ISA) had more adrenoceptors than control subjects [44] and that the adenylate cyclase activation by the $\beta$-adrenoceptor agonist isoprenaline is also enhanced in this situation [45]. The ISA is therefore an important criterion for the therapeutical usefulness of BBs in heart failure patients.

The cardiac effects of BBs are varying in human cardiac tissue. The cardiodepressant effects of beta-blocker may not correlate with its $\beta 1$-selectivity but result from the combined effects of $\beta 1$-selectivity, intrinsic sympathomimetic properties and inverse agonism [46].

The reason for the cardioprotective effects of carvedilol in ANTinduced cardiomyopathy (CMP) may occur through the potent 
antioxidant activity of carvedilol. Both carvedilol and its metabolites were shown to have antioxidant effects [47]. It was reported that free oxygen radicals in failing heart were reduced by administration of carvedilol [48]. This antioxidant activity of carvedilol is attributed to its ability to chelate free iron [49], which is widely implicated in enhancing the free radical-mediated toxicity caused by doxorubicin.

However, other possible mechanisms may involve the protection of carvedilol. Sarcoplasmic reticulum $\mathrm{Ca}^{2+}$-ATPase (SERCA2) may be another key factor in ANT-induced cardiotoxicity. Doxorubicin causes down-regulation of SERCA2 messenger RNA in animals with cardiac dysfunction [50]. In addition, ANT has been noted to stimulate the release of $\mathrm{Ca}^{2+}$ in cardiomyocytes [51]. Treatment of carvedilol is also associated with inhibition of apoptotic signaling pathways [52]. Because apoptosis plays a highly significant role in ANT-induced CMP [53], the antiapoptotic properties of carvedilol could be another important factor in protection from ANT-induced CMP.

A study by Wawaimuli et al. [28] was designed to test the effectiveness of carvedilol, against Dox-induced cardiotoxicity and nephrotoxicity in rats. Carvedilol down-regulated matrix metalloproteinase-2 expression in the heart, increased nephrin expression in the kidney, and attenuated the increased protein expression of NADPH oxidase subunits in heart and kidney. Moreover, carvedilol reduced myocardial and renal apoptosis and improved the histopathological changes in heart and kidney induced by Dox.

The prominent cardioprotective effects of the third-generation beta-blocker nebivolol against anthracycline-induced cardiotoxicity, using the model of an isolated perfused rat heart, were examined by de Nigris et al. [25] Sprague-Dawley rats were treated with Dox and daunorubicin in combination with nebivolol or carvedilol. The cotreatment of beta-blockers and anthracyclines had reduced the release of GSSG and GSH. A more significant reduction was shown with nebivolol than with carvedilol. Also, significant reductions of CK and troponin $\mathrm{T}$ activities were observed when the hearts were treated with nebivolol. At the same time, GSHPx, MnSOD, and nitrite/nitrate release were increased after the co-treatment. Three cardiac parameters have been used to evaluate the cardiac toxicity of both anthracyclines and beta-blocker-anthracycline combinations. The left ventricular pressure developed under a constant perfusion pressure (LVDP), then the variation rates of this parameter were observed during contractility (LV/dt)max and relaxation $\mathrm{LV}(\mathrm{dP} / \mathrm{dt})$ min. Nebivolol in combination with anthracyclines had exerted the most significant protection of heart tissue.

It has been demonstrated that nebivolol affected vasodilatation through NO production, stimulated NO release, enhanced NO bioavailability and prevented NO deactivation [54]. A study by Angelo et al. [54] demonstrated that nebivolol stimulates NO production in the heart. This action of nebivolol is exerted via a signaling pathway starting from the activation of $\beta 3$-adrenergic receptors and leading to overexpression of inducible $\mathrm{NO}$ synthase.

Nebivolol, a last-generation beta blocker, which is a selective $\beta 1$ adrenergic receptor antagonist, it leads to (i) an increase in the renal NO excretion (ii) a significant increase in the renal plasma flow and glomerular filtration rate (GFR)[56], (iii) suppression of the reninangiotensin aldosterone system and inhibition angiotensin II[56,57], and reduces endothelin-1 [58], and (iv) has an antioxidant effect [59].

Alexandros et al. [60] compared the efficacy of nebivolol versus carvedilol on left ventricular (LV) function and exercise capacity in patients with nonischemic dilated cardiomyopathy (NIDC). Both nebivolol and carvedilol appear relatively safe, with beneficial effects on LV systolic and diastolic function as well as exercise capacity in patients with NIDC after 12 months' treatment. However, carvedilol exhibits more favorable effects on LV function than does nebivolol.

\section{Conclusions}

A coadministration of either carvedilol or nebivolol with doxorubicin was able to ameliorate up to almost contradict doxorubicin-induced myocardial injury, by a mechanism not related to their $\beta$-adrenergic receptor antagonism. Also, they improved glomerular filtration disturbance and renal tubular damage with upper hand for nebivolol. So, either drug, especially nebivolol, must merit serious consideration as an adjunctive therapy to protect against cardiotoxicity and nephrotoxicity commonly encountered with doxorubicin treatment.

Further investigations are needed to explore the possible mechanisms of the protective actions of carvedilol and nebivolol.

\section{References}

1. Floyd J, Mirza I, Sachs B, Perry MC (2006) Hepatotoxicity of chemotherapy. Semin Oncol 33: 50-67.

2. Stebbing J, Jones A, Barrett-Lee $P(2008)$ Cardiotoxicity and anthracyclines Breast Cancer Res Treat 107: 451-453.

3. Priestman T (2008) Cancer chemotherapy in clinical practice. Springer- Verlag London, UK press.

4. Kocak G, Erbil KM, Ozdemir I, Aydemir S, Sunar B (2003) The protective effect of melatonin on adriamycin-induced acute cardiac injury. Can J Cardiol 19: 535541.

5. Banerjee HN, Verma M (2008) Application of nanotechnology in cancer Technol Cancer Res T 7: 149-154.

6. Fadillioglu E, Erdogan H, Sogut S, Kuku I (2003) Protective effects of erdosteine against doxorubicin-induced cardiomyopathy in rats. J Appl Toxicol 23: 71-74.

7. Quiles JL, Huertas JR, Battino M, Mataix J, Ramírez-Tortosa MC (2002) Antioxidant nutrients and adriamycin toxicity. Toxicol 180: 79-95.

8. Stolarska M, Mlynarski W, Zalewska-Szewczyk B, Bodalski J (2006) Cytoprotective effect of amifostine in the treatment of childhood neoplastic diseases-- a clinical study including the pharmacoeconomic analysis. Pharmacol Rep 58: 30-34.

9. Yeh YC, Lai HC, Ting CT, Lee WL, Wang LC, et al. (2007) Protection by doxycycline against doxorubicin induced oxidative stress and apoptosis in mouse testes. Biochem Pharmacol 74: 969-980.

10. Liu LL, Li QX, Xia L, Li J, Shao L (2007) Differential effects of dihydropyridine calcium antagonists on doxorubicin-induced nephrotoxicity in rats. Toxicology 231: $81-90$.

11. Fu P, Arcasoy MO (2007) Erytthropoietin protects cardiac myocytes against anthracycline-induced apoptosis. Biochem Biophys Res Commun 354: 372 378 .

12. Sakaguchi H, Kodama A, Tomonari M, Ando Y, Tabuchi M, et al. (2008) Preadministration of docetaxel protects against adriamycin-induced cardiotoxicity. Breast Cancer Res Treat 109: 443-450.

13. Injac R, Perse M, Cerne M, Potocnik N, Radic N, et al. (2009) Protective effects of fullerenol $\mathrm{C} 60(\mathrm{OH}) 24$ against doxorubicin-induced cardiotoxicity and hepatotoxicity in rats with colorectal cancer. Biomaterials 30: 1184-1196.

14. Injac R, Boskovic M, Perse M, Koprivec-Furlan E, Cerar A, et al. (2008) Acute doxorubicin nephrotoxicity in rats with malignant neoplasm can be successfully treated with fullerenol $\mathrm{C} 60(\mathrm{OH}) 24$ via suppression of oxidative stress. Pharmacol Rep 60: 742-749.

15. Injac R, Strukelj B (2008) Recent advances in protection against doxorubicininduced toxicity. Technol Cancer Res Treat 7: 497-516.

16. Wollert KC, Drexler H (2002) Carvedilol prospective randomized cumulative survival (COPERNICUS) trial: carvedilol as the sun and center of the betablocker world? Circulation 106: 2164-2166. 
Citation: Shafik AN, Khodeir MM, Fadel MS (2011) Animal study of Anthracycline-induced Cardiotoxicity and Nephrotoxicity and Evaluation of Protective Agents. J Cancer Sci Ther 3: 096-103. doi:10.4172/1948-5956.1000068

17. Flather MD, Shibata MC Coats AJ, Van Veldhuisen DJ, Parkhomenko A, et al. (2005) Randomized trial to determine the effect of nebivolol on mortality and cardiovascular hospital admission in elderly patients with heart failure (SENIORS). Eur Heart J 26: 215-225.

18. Cheng J, Kamiya K, Kodama I (2001) Carvedilol: Molecular and cellular basis for its multifaceted therapeutic potential. Cardiovasc Drug Rev 19: 152-171.

19. Dulin B, Abraham WT (2004) Pharmacology of carvedilol Am J Cardiol 93: 3-6.

20. Nodari S, Metra M, Dei Cas L (2003) $\beta$-Blocker treatment of patients with diastolic heart failure and arterial hypertension. A prospective, randomized, comparison of the long-term effects of atenolol vs. nebivolol. Eur J Heart Fail 5: 621-627.

21. Maffei A, Aretini A, Vecchione C, Poulet R, Marino G, et al. (2006) Nebivolol induces nitric oxide production through eNOS phosphorylation. Am J Hyperten 19: $579-586$

22. Paz Y, Gurevitch J, Frolkis I, Matsa M, Kramer A, et al. (1998) Effects of an angiotensin II antagonist on ischemic and nonischemic isolated rat hearts. Ann Thorac Surg 65: 474-479.

23. Kotolová H, Kollár M, Jaro O (2006) Carvedilol Protects against Cyclosporine Nephropathy in Rats. Acta Vet Brno 75: 85-89.

24. Toprak O, Cirit M, Tanrisev M, Yazici C, Canoz O, et al. (2008) Preventive effect of nebivolol on contrast-induced nephropathy in rats. Nephrol Dial Transplant 23: 853-859.

25. de Nigris F, Rienzo M, Schiano C, Fiorito C, Casamassimi A (2008) Prominent cardioprotective effects of third generation beta blocker nebivolol against anthracycline-induced cardiotoxicity using the model of isolated perfused rat heart. Eur J Cancer 44: 334-340.

26. Menry RJ (1974) Clinical principles and techniques, 2nd Edition, Harper and row, $525 p$

27. Reinhold JG (1953) Total protein, albumin and globulin. Stand Methods Clin Chem 1:88.

28. Wawaimuli A, Kenichi W, Punniyakoti TV, Meilei M, Rajarajan AT, et al. (2010) Protective effect of carvedilol on daunorubicin-induced cardiotoxicity and nephrotoxicity in rats. Toxicology 274: 18-26.

29. StuĖcker O, Vicaut E, Villereal MC, Ropars C, Teisseire BP, et al. (1985) Coronary response to large decreases of haemoglobin-O2 affinity in isolated rat heart. Am J Physiol 249: H1224-1227.

30. Singal PK, Iliskovic N (1989) Doxorubicin-Induced Cardiomyopathy. The New England Journal of Medicine 339: 900-905.

31. Robert J (2007) Preclinical assessment of anthracycline cardiotoxicity in laboratory animals: Predictiveness and pitfalls. Cell Biol Toxicol 23: 27-37.

32. Hideo K, Shiho F, Yukihiro K, Akira K, Hiroyuki S, et al. (1991) Alterations of intracellular calcium homeostasis and myocardial energetics in acute adriamycin-induced heart failure. J Cardiovasc Pharmacol 18: 437-444.

33. Nagami K, Yoshikawa T, Suzuki M, Wainai Y, Anzai T, et al. (1997) Abnorma beta-adrenergic transmembrane signaling in rabbits with adriamycin-induced cardiomyopathy. Jpn Circ J 61: 249-255

34. de Nigris F, Rienzo M, Schiano C, Fiorito C, Casamassimi A, et al. (2008) Prominent cardioprotective effects of third generation beta blocker nebivolol against anthracycline-induced cardiotoxicity using the model of isolated perfused rat heart. Eur J Cancer 44: 334-340.

35. Oliveira PJ, Bjork JA, Santos MS, Leino RL, Froberg MK, et al. (2004) Carvedilol-mediated antioxidant protection against doxorubicin-induced cardiac mitochondrial toxicity. Toxicol Appl Pharmacol 200: 159-168.

36. Jovanovic D, Djukanovic I, Susic D, Funduk G, Jovanovic Z, et al. (1996) The effect of captopril on the development of adriamycin nephropathy in rats with spontaneous arterial hypertension. Srp Arh Celok Lek 124: 47-49.

37. Lebrecht D, Setzer B, Rohrbach R, Walker UA (2004) Mitochondrial DNA and its respiratory chain products are defective in doxorubicin nephrosis. Nephrol Dial Transplant 19: 329-336.

38. Luft FC (2006) Doxorubicin toxicity in the Iron Age. J Mol Med 84: 529-531.
39. Panjrath GS, Patel V, Valdiviezo Cl, Narula N, Narula J, et al. (2007) Potentiation of doxorubicin cardiotoxicity by iron loading in a rodent model. J Am Coll Cardio 49: $2457-2464$

40. Washio M, Nanishi F, Okuda S, Onoyama K, Fujishima M (1994) Alpha tocopherol improves focal glomerulosclerosis in rats with Adriamycin-induced progressive renal failure. Nephron 68: 347-352.

41. Brodde OE (1991) $\beta 1$ and $\beta 2$-adrenoceptors in the human heart: Properties function, and alterations in chronic heart failure. Pharmacol Rev 43: 203-242.

42. Stoschitzky K, SakotnikA, Lercher P, Zweiker R, Maier R, et al. (1999) Influence of beta-blockers on melatonin release. Eur J Clin Pharmacol 55: 111-115.

43. Carreira RS, Monteiro P, Gon Alves LM, Providencia LA (2006) Carvedilol: just another Beta-blocker or a powerful cardioprotector? Cardiovasc Hematol Disord Drug Targets 6: 257-266.

44. Motomura S, Deighton NM, Zerkowski HR, Doetsch N, Michel MC, et al. (1990) Chronic beta1-adrenoceptor antagonist treatment sensitizes beta2 adrenoceptors, but desensitizes M2-muscarinic receptors in the human right atrium. Br J Pharmacol 101: 363-369.

45. Trochu JN, Erfanian M, Khandoudi N, Baron O, Bril A, et al. (1999) Carvedilo produces contractile effects different from those of bucindolol in human atria. Circulation. 100 Suppl: 1-439.

46. Klara B, Andreas B, Birgit B, Mehlhorn U , Robert HGS (2001) Nebivolol, bucindolol, metoprolol and carvedilol are devoid of intrinsic sympathomimetic activity in human myocardium. Br J Pharmacol 133: 1330-1338.

47. Abreu RM, Santos DJ, Moreno AJ (2000) Effects of carvedilol and its analog BM-910228 on mitochondrial function and oxidative stress. J Pharmacol Exp Ther 295: 1022-1030.

48. Ide T, Tsutsui $\mathrm{H}$, Kinugawa S, Utsumi $\mathrm{H}$, Kang D, et al. (1999) Mitochondria electron transport complex I is a potential source of oxygen free radicals in the failing myocardium. Circ Res 85: 357-363.

49. Noguchi N, Nishino K, Niki E (2000) Antioxidant action of the antihypertensive drug, carvedilol, against lipid peroxidation. Biochem Pharmacol 59: 1069-1076.

50. Arai M, Tomaru K, Takizawa T, Sekiguchi K, Yokoyama T, et al. (1998) Sarcoplasmic reticulum genes are selectively down-regulated in cardiomyopathy produced by doxorubicin in rabbits. J Mol Cell Cardiol 30: 243-254.

51. Boucek RJ Jr, Buck SH, Scott F, Oquist NL, Fleischer S, et al. (1993) Anthracycline-induced tension in permeabilized cardiac fibers: evidence for the activation of the calcium release channel of sarcoplasmic reticulum. J Mol Cell Cardiol 25: 249-259

52. Spallarossa P, Garibaldi S, Altieri P, Fabbi P, Manca V, et al. (2004) Carvedilol prevents doxorubicin-induced free radical release and apoptosis in cardiomyocytes in vitro. J Mol Cell Cardiol 37: 837-846.

53. Muller I, Niethammer D, Bruchelt G (1998) Anthracycline-derived chemotherapeutics in apoptosis and free radical cytotoxicity. Int $\mathrm{J} \mathrm{Mol} \mathrm{Med} 1$ : 491-494.

54. Kakoki M, Hirata Y, Hayakawa H, Nishimatsu H, Suzuki Y, et al. (1999) Effects of vasodilatory betaadrenoceptor antagonists on endothelium-derived nitric oxide release in rat kidney. Hypertension 33: 467-471.

55. Maffei A, Di Pardo A, Carangi R, Carullo P, Poulet R, et al. (2007) Nebivolo Induces Nitric Oxide Release in the Heart through Inducible Nitric Oxide Synthase Activation. Hypertension 50: 652-656.

56. Greven J, Gabriels G (2000) Effect of nebivolol, a novel beta 1-selective adrenoceptor antagonist with vasodilating properties, on kidney function Arzneimittelforschung 50: 973-979.

57. Blumenfeld JD, Sealey JE, Mann SJ, Bragat A, Marion R, et al. (1999) Betaadrenergic receptor blockade as a therapeutic approach for suppressing the reninangiotensin- aldosterone system in normotensive and hypertensive subjects. Am J Hypertens 12: 451-459.

58. Brehm BR, Bertsch D, von Fallois J, Wolf SC (2000) Beta-blockers of the third generation inhibit endothelin-1 liberation, mRNA production and proliferation of human coronary smooth muscle and endothelial cells. J Cardiovasc Pharmaco 36: S401-S403. 
Citation: Shafik AN, Khodeir MM, Fadel MS (2011) Animal study of Anthracycline-induced Cardiotoxicity and Nephrotoxicity and Evaluation of Protective Agents. J Cancer Sci Ther 3: 096-103. doi:10.4172/1948-5956.1000068

59. Mason RP, Kubant R, Jacob RF, Walter MF, Boychuk B, et al. (2006) Effect of nebivolol on endothelial $\mathrm{NO}$ and peroxynitrite release in hypertensive animals: Role of antioxidant activity. J Cardiovasc Pharmacol 48: 862-869.
60. Patrianakos AP, Parthenakis FI, Mavrakis HE, Diakakis GF, Chlouverakis $\mathrm{Gl}$, et al. (2005) Comparative Efficacy of Nebivolol Versus Carvedilol on Lef Ventricular Function and Exercise Capacity in Patients With Nonischemic Dilated Cardiomyopathy. A 12-month study. Am Heart J 150: 985 